Revista de

Estudios

Kantianos

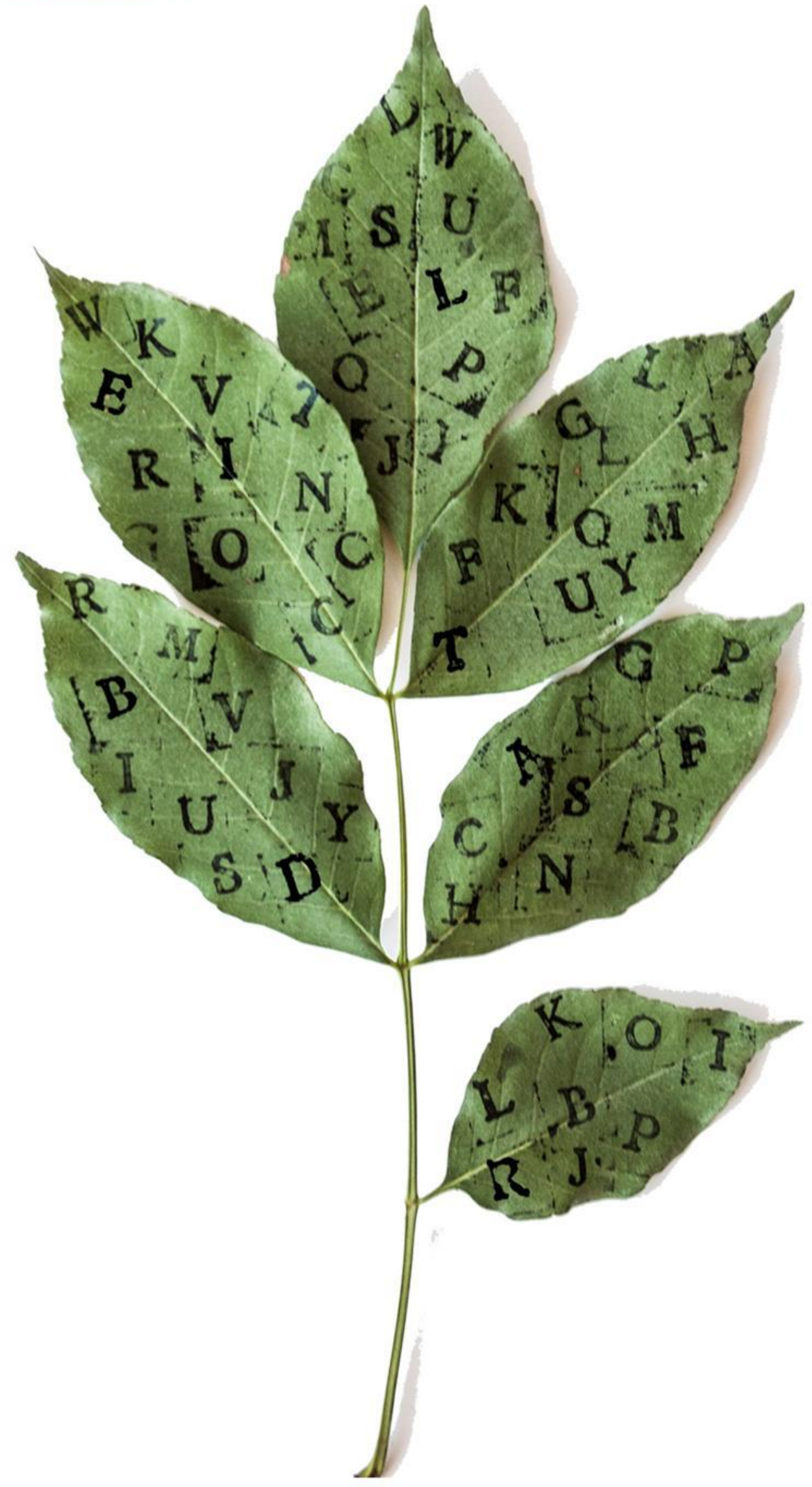




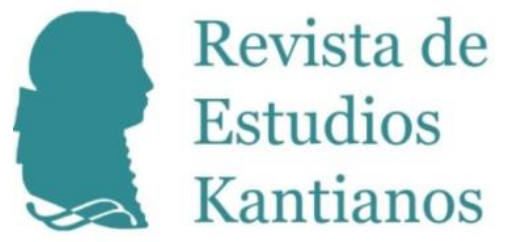




\title{
Revista de Estudios Kantianos
}

\author{
Publicación internacional de la Sociedad de Estudios Kantianos en Lengua Española \\ Internationale Zeitschrift der Gesellschaft für Kant-Studien in Spanischer Sprache \\ International Journal of the Society of Kantian Studies in the Spanish Language
}

\section{Dirección}

Fernando Moledo, FernUniversität in Hagen

fernando.moledo@fernuni-hagen.de

Hernán Pringe, CONICET-Universidad de Buenos Aires/

Universidad Diego Portales, Santiago de Chile

hpringe@gmail.com

\section{Secretario de edición}

Óscar Cubo Ugarte, Universitat de València

oscar.cubo@uv.es

\section{Secretaria de calidad}

Alba Jiménez Rodríguez, Universidad Complutense de Madrid

albjim04@ucm.es

\section{Editores científicos}

Jacinto Rivera de Rosales, UNED, Madrid

Claudia Jáuregui, Universidad de Buenos Aires

Vicente Durán, Pontificia Universidad Javeriana, Bogotá

Julio del Valle, Pontificia Universidad Católica del Perú, Lima

Jesús Conill, Universitat de València

Gustavo Leyva, Universidad Autónoma de México, México D. F.

María Xesús Vázquez Lobeiras, Universidade de Santiago de Compostela

Wilson Herrera, Universidad del Rosario, Bogotá

Pablo Oyarzun, Universidad de Chile, Santiago de Chile

Paula Órdenes Azúa, Universität Heidelberg 


\section{Comité científico}

Juan Arana, Universidad de Sevilla

Reinhardt Brandt, Philipps-Universität Marburg

Mario Caimi, Universidad de Buenos Aires

Monique Castillo, Université de Paris-Est

Adela Cortina, Universitat de València

Bernd Dörflinger, Universität Trier

Norbert Fischer, Universität Eichstätt-Ingolstadt

Miguel Giusti, Pontificia Universidad Católica del Perú

Dulce María Granja, Universidad Nacional Autónoma de México

Christian Hamm, Universidad Federal de Santa María, Brasil

Dietmar Heidemann, Université du Luxembourg

Otfried Höffe, Universität Tübingen

Claudio La Rocca, Università degli Studi di Genova

Juan Manuel Navarro Cordón, Universidad Complutense, Madrid

Carlos Pereda, Universidad Nacional Autónoma de México

Gustavo Pereira, Universidad de la República, Uruguay

Ubirajara Rancan de Azevedo, Universidade Estadual Paulista, Brasil

Margit Ruffing, Johannes Gutenberg-Universität Mainz

Gustavo Sarmiento, Universidad Simón Bolívar, Venezuela

Sergio Sevilla, Universitat de València

Roberto Torretti, Universidad Diego Portales, Santiago de Chile

Violetta Waibel, Universität Wien

Howard Williams, University of Aberystwyth

Allen W. Wood, Indiana University

Diseño, revisión de estilo, corrector y maqueta

Josefa Ros Velasco, Harvard University, Cambridge (MA)

\section{Entidades colaboradoras}

Sociedad de Estudios Kantianos en Lengua Española (SEKLE)

Departament de Filosofia de la Universitat de València

Instituto de Humanidades, Universidad Diego Portales

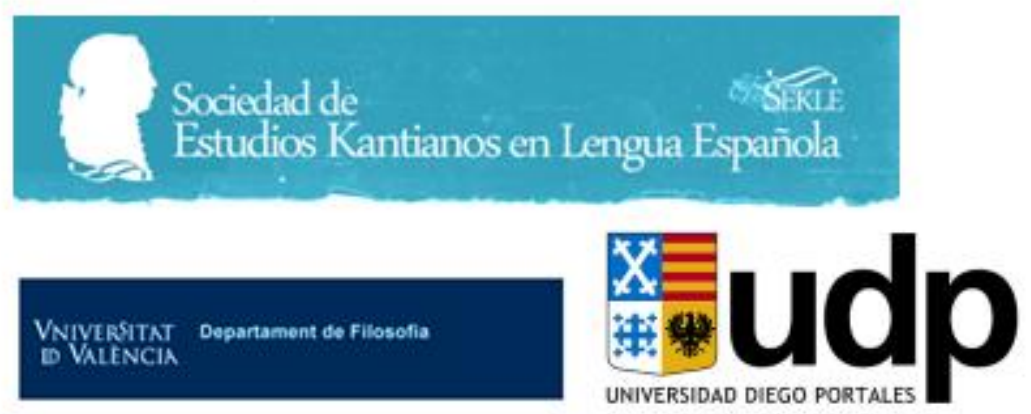




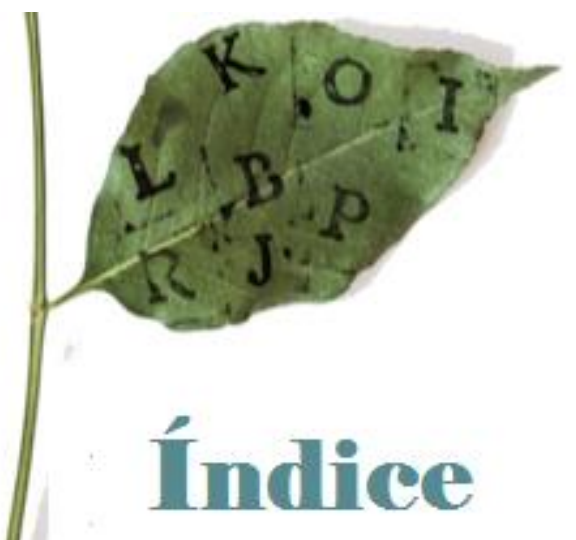

\section{Artículos}

1 El concepto teórico de libertad según Kant y la tradición de la libertas spontaneitatis

Thomas Sören Hoffmann

DOI 10.7203/REK.4.1.14849

21 From the unity of sensible intuition to the sensible unity of intuition. Revisiting the proof-structure of Kant's B-Deduction argument Adriano Perin

DOI: 10.7203/REK.4.1.12644

44 The Principle of the Transcendental Deduction. The First Section of the Deduction of the Pure Concepts of the Understanding Rudolf Meer DOI: 10.7203/REK.4.1.14339

63 ¿Por qué son — según Kant — imposibles las hipótesis en matemática?

Reyna Fortes

DOI: 10.7203/REK.4.1.12777 
90 El reino de los fines y la comunidad ética. Acerca de la dimensión intersubjetiva de la ética kantiana

Ileana Beade

DOI: 10.7203/REK.4.1.12775

\section{Recensiones}

113 Mario Caimi: Kant's B Deduction. Newcastle upon Tyne, Cambridge Scholars Publishing, 2014, 140 pp. ISBN (13): 978-1-4438-6537-1 Paola Rumore

DOI: 10.7203/REK.4.1.14280

121 Gustavo Leyva, Álvaro Peláez y Pedro Stepanenko (Eds.): Los rostros de la razón: Immanuel Kant desde Hispanoamérica (3 volúmenes). México, UAM-Cuajimalpa/Anthropos, 2018, 727 pp. ISBN (UAM): 978-607-28-1348-9/ISBN (Anthropos): 978-8416421-91-6

Francisco Javier Iracheta Fernández.

DOI: 10.7203/REK.4.1.14135

134 Ileana Beade: La libertad y el orden en la filosofía jurídica kantiana. Rosario, Fhumyar Ediciones, 2017, 310 pp. ISBN: 978-987-3638-169

Marilín Gómez.

DOI: $10.7203 /$ REK.4.1.14266

138 Christian Krijnen (Ed.): Metaphysics of Freedom? Kant's Concept of Cosmological Freedom in Historical and Systematic Perspective.

Leiden/Boston, Brill (Critical Studies in German Idealism, vol. 23), 2018, 221 pp. ISBN: 978-90-40-38377-7

Jacinto Páez.

DOI: 10.7203/REK.4.1.14337 


\section{Traducción}

145 Salomon Maimon frente a la filosofía trascendental. Traducción de algunas cartas sobre su filosofía teórica

David Hereza Modrego

DOI: 10.7203/REK.4.1.13956

\section{Informes}

173 Informe del IV Congreso Internacional de la SEKLE Alejandra Baher

Luciana Martínez

DOI: 10.7203/REK.4.1.14281

178 Ata do Simpósio Internacional de Kant a Hegel Agemir Bavaresco

Jair Tauchen

Evandro Pontel

DOI: 10.7203/REK.4.1.14269

\section{Eventos y normas para autores}

185 Normas para autores 


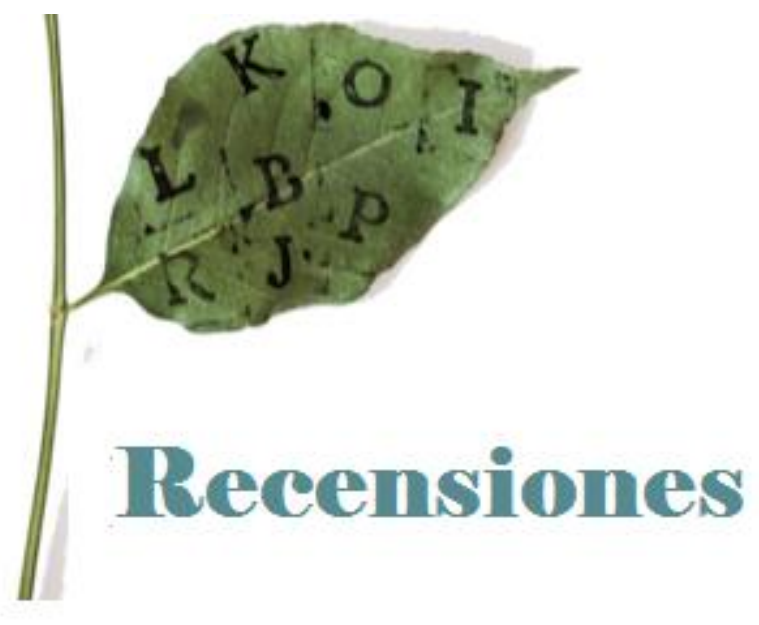




\title{
Christian Krijnen (Ed.): Metaphysics of Freedom? Kant's Concept of Cosmological Freedom in Historical and Systematic Perspective. Leiden/Boston, Brill (Critical Studies in German Idealism, vol. 23), 2018, 221 pp. ISBN: 978-90-40-38377-7
}

\author{
JACINTO PÁEZ ${ }^{1}$
}

En un conocido pasaje de Los Progresos de la Metafísica, Kant advierte que la realidad de la libertad es, junto con la idealidad de las representaciones de espacio y tiempo, el sostén sobre el cual descansa la totalidad de la metafísica. Además de ocupar este rol arquitectónico, el concepto kantiano de libertad es complejo y polisémico, tal como lo muestra la coexistencia, en su teoría, de sus especificaciones como libertad transcendental —o cosmológica, libertad práctica, o arbitrio - . Al estudio de la primera variante mencionada está dirigido el libro editado por Christian Krijnen. El libro ofrece al lector tanto reconstrucciones sistemáticas de la teoría kantiana como así también el desarrollo de una perspectiva histórica, tanto en lo que refiere a la larga tradición filosófica desde la cual Kant parte, como a su proyección en los diversos sistemas del idealismo alemán y del neokantismo. El lector verá que es esta perspectiva histórica la que determina el orden de los capítulos del volumen y a la cual nos ceñiremos en nuestra síntesis del mismo.

El libro comienza acertadamente con el ensayo "Free Will in Antiquity and in Kant", escrito por Michael Forster. Para el autor, al concepto kantiano de libertad subyace la formulación originaria del pensamiento griego. Por tal motivo, dedica gran parte de su ensayo a realizar una genealogía del modelo que ha devenido estándar del pensamiento occidental en torno a la libertad. Este modelo, que define a la libertad como capacidad de elección entre cursos de acción alternativos, encuentra su expresión paradigmática en la conjunción, no necesariamente compatible, de las premisas estoicas y aristotélicas realizadas por Alejandro de Afrodisia. Independientemente del interés en sí de esta genealogía, Forster sostiene que

${ }^{1}$ Universidad Diego Portales. Contacto: jacintopaez.88@gmail.com. 
la misma permite comprender la distinción de Kant entre una libertad como espontaneidad y una libertad como autonomía en tanto reflejo del modelo antiguo. El aspecto positivo de esta propuesta es que disuelve el llamado problema de la imputabilidad en la filosofía práctica kantiana, pero lo hace con el costo de proyectar sobre ella las oscuridades argumentales de las fuentes griegas; oscuridades que, para el autor, la filosofía de Kant tampoco consigue clarificar de modo satisfactorio.

El segundo capítulo corre a cuenta de Gábor Boros y lleva por título "Freedom in Nature, Freedom of the Mind". El tema del capítulo es el concepto cosmológico de libertad de Spinoza. Boros señala que coexisten dos definiciones de la libertad en la Ética: existencia como siguiendo de la propia esencia (E1, D7) y como el amor constante y eterno de Dios (E5, P36, escolio). La estrategia del autor para interpretar su coexistencia consiste en exponerlas como una apropiación de dos antecedentes específicos: el voluntarismo de Descartes y el concepto de amor divino de León Hebreo. La posición propia de Spinoza aparece así como la combinación del proyecto cartesiano de una metafísica orientada hacia una imagen científica del mundo y una implementación de tal proyecto que recoge elementos del neoplatonismo judío. En cualquier caso, el capítulo ofrece una reconstrucción detallada de los argumentos y las críticas que llevaron a Spinoza a ofrecer un concepto original de libertad que se coloca en la vereda contraria del concepto cosmológico de libertad de Kant.

Thomas Hoffmann escribe "Kants theoretischer Freiheit und die Tradition der "libertas spontaneitatis"'. La propuesta del capítulo consiste en reconstruir la constelación filosófica que oficia como punto de partida del concepto de libertad de Kant. Así, si bien Hoffmann reconoce el valor de la genealogía trazada por Forster previamente, también considera que el debate moderno adquiere una forma propia e irreductible a los modelos de los antiguos. Evidentemente, los autores modernos ya no comparten los presupuestos de la ontología aristotélica. A través de una serie de ejemplos históricos el autor expone el desarrollo racionalista de la idea de libertad como espontaneidad que en el período queda atada a la filosofía de Leibniz. Es precisamente la posición leibniziana el punto de partida de Kant en su Nova dilucidatio de 1755, aunque, ya en esta obra primeriza, la posición de Kant presenta matices propios. La última sección del capítulo considera el resultado de la 'tercera antinomia'. Hoffmann afirma que el verdadero 
rendimiento de Kant es haber alcanzado una noción de libertad como idea racional en su autorrealización y, entonces, un concepto de libertad que ya no pertenece exclusivamente al mundo de los entes.

El capítulo escrito por Klaus Erich Kaehler, "The Freedom of the Monad and the Subject of Freedom", propone una confrontación entre los conceptos de libertad de Leibniz y Kant. El concepto de libertad es el resultado sistemático de la construcción metafísica de Leibniz, por lo cual el Kaehler ofrece una síntesis muy compacta de la metafísica objetiva de aquel. La libertad es definida por Lebiniz como espontaneidad inteligente, la cual, en su grado máximo, se presenta en Dios. La libertad de la mónada creada no puede ser caracterizada como auto-creación - pero sí hay un concepto de libertad en analogía a la libertad creadora de Dios: como compromiso con una sujeción de la voluntad a la afirmación de una aprehensión racional- Kant mantiene el tipo de definición lebiniziana en la medida en que afirma el nexo entre razón y libertad pero desde la perspectiva de una auto-relación de la razón. La última sección del texto intenta mostrar cómo los argumentos kantianos desarrollan esta perspectiva sin recaer en un compromiso con una metafísica objetiva.

Heiner Klemme escribe "Das Problem der transzendetalen Freiheit und seine Lösung: Kant versus Wolff". Como indica el título de modo explícito, Klemme presenta las peculiaridades del concepto kantiano de libertad transcendental a partir de una contraposición con la Deutschen Metaphysik de Wolff y con textos de Christian Garve. Klemme ofrece una amplia cantidad de referencias al tratamiento de la libertad racionalista en el marco de la metafísica especial (cosmología y teología) y también en el de la psicología empírica. En el caso de Garve, el tema de la libertad se presenta a través de una discusión de la antinomia entre fatalistas e indiferentistas que anticipa la antinomia kantiana - pero en un plano moral, no metafísico-. El planteo de Garve se desenvuelve exclusivamente en terreno de la razón práctica y, por ende, sin explicar cómo ella es teóricamente posible. Kant retorna con este concepto al terreno de la cosmología, pero, a diferencia de Wolff, prepara su respuesta provisto de la diferencia entre entendimiento y razón. La última sección del ensayo reconstruye los argumentos que Kant elabora a partir de este nuevo punto de vista. Como conclusión histórica del texto, podemos decir que el legado de Garve es la formulación del problema del cual el concepto de libertad transcendental es la respuesta. 
Stephan Zimmermann redacta el séptimo capítulo, "Kant on 'Practical Freedom' and Its Transcendental Possibility". Se trata de un largo estudio, exclusivamente dedicado a Kant, en el cual el lector encuentra como principal característica un análisis textual muy detallado. El principal problema interpretativo es la homonimia en el concepto kantiano, que hace necesario establecer una serie de distinciones terminológicas: libertad transcendental; libertad práctica; libertad empírica; arbitrio sensible e intelectual (según la Metafísica Pölitz); voluntad (Wille) y poder de elección (Willkür). Estos matices, sin embargo, se articulan siempre en función de una capacidad causal que tiene dos momentos, un momento determinativo y otro momento ejecutivo. Esta distinción es, en general, la clave hermenéutica utilizada por Zimmerman. El texto, de todas maneras, no registra solamente un plano descriptivo, sino que intenta mostrar que el problema más serio en torno al concepto de libertad es aquel de su posibilidad transcendental o el de su compatibilidad con la segunda analogía de la experiencia (posibilidad de la libertad en sentido empírico), lo que motiva al autor a abandonar la discusión del concepto de libertad en sentido moral para concentrarse en la discusión del determinismo natural, es decir, para regresar al problema cosmológico.

El capítulo octavo se titula "Absolute Spontaneity and SelfDetermination: The Fact of Reason and the Categories of Freedom", escrito por Martin Bunte. El autor continúa con la tónica del capítulo previo, ofreciendo comentarios extensos de pasajes tanto de la Crítica de la razón pura como de la Crítica de la razón práctica, pero moviéndose por fuera del tópico de la libertad cosmológica, en la medida en que la mayor parte de su preocupación concierne a la teoría del faktum de la razón. Destaca, en el capítulo, la explicación de la construcción lógica de la tabla de las categorías de la libertad, uno de los pasajes más oscuros de la segunda crítica, a partir de la idea de auto-determinación. Así, esta idea forma, aunque desde una óptica diversa, el núcleo teórico de ambas críticas.

Con el texto de Marina Bykova, "Kant's Problem with Freedom and Fichte's Response to the Challenge", el volumen pasa a considerar el legado de Kant. El problema al cual se refiere el título es el del carácter autooriginario de la acción libre, pues Kant no ofrece una prueba o explicación de su actualidad, sino solamente de su posibilidad. Para la autora, la virtud de Fichte consiste en ofrecer una teoría de la realidad de la libertad cosmológica a través del principio supremo de la filosofía: la auto-posición del yo. En este 
sentido, la autora defiende, a través de un comentario de la Doctrina de la Ciencia y del Sistema de Ética, que Fichte, frente a Kant, es un innovador en la teoría de la libertad en sentido cosmológico.

Faustino Fabianelli se encarga del capítulo décimo, titulado "Sameness and Otherness in the Free Principle of Philosophy: Fichte's Wissenschaftslehre in Comparison to Hegel's Science of Logic". El autor se embarca en una discusión teórica entre la filosofía de Fichte (interpretada como formulación de un pensamiento sobre lo absoluto en clave heterotética - un término técnico tomado del filósofo neokantiano Heinrich Rickert) - y la de Hegel (interpretada en términos de dialéctica). En el caso de Fichte, la libertad del principio de la Doctrina de la Ciencia es una libertad analógica, pues es la libertad de una imagen que se considera a sí misma como imagen del absoluto, mientras que, según el principio de la dialéctica, tal libertad analógica no es una libertad plena, pues se define gracias a su contrapartida, el absoluto, que permanece externo. Fabianelli se muestra partidario de la solución fichteana en la medida en que no reduce la realidad al poder del concepto, aunque la cuestión lamentablemente no es saldada con claridad en el texto.

El capítulo onceavo fue escrito por Christian Krijnen y se titula "Kant's Conception of Cosmological Freedom and its Metaphysical Legacy". La particularidad del ensayo consiste en considerar el concepto kantiano en función de su legado problemático. Como ha quedado claro para el lector a esta altura, el concepto kantiano revoluciona el modo de pensar la libertad sin dejar, por otra parte, de tener como lastre determinaciones conceptuales propias de la tradición metafísica. Krijnen analiza dos actitudes postkantianas frente a estas presuposiciones: el rechazo de Hegel y la transformación neokantiana. En el caso de Hegel, se niega que la libertad tenga el carácter de un poder causal, en el cuál ambos miembros de la relación solo están enlazados de modo externo. Para Hegel, aquello que es libre es el concepto y la libertad es auto-relación. Pero quizá lo más interesante para el lector hispano (probablemente también para el anglófono) sea la referencia al neokantismo, específicamente, a Bruno Bauch, el cual define en función de una de sus afirmaciones centrales, a saber, la concepción teórica de la normatividad. En la teoría de la acción de Bauch se explicita un modelo de causalidad que integra, a través del concepto de subjetividad concreta, tanto la causalidad natural como la causalidad por libertad. 
Desde su mismo título, "Hegel's Concept of Recognition as the Solution to Kant's Third Antinomy”, el capítulo escrito por Arthur Kok plantea un argumento original para reconstruir la crítica de Hegel al concepto de libertad de Kant. El concepto de reconocimiento es la clave para comprender la posición de Hegel con respecto a la distinción entre fenómeno y cosa en sí y, por ende, para abordar el problema de la tercera antinomia kantiana, solo que ofreciendo una solución diferente al idealismo transcendental. Luego de establecer algunas críticas preliminares al idealismo transcendental kantiano, el autor introduce el concepto de reconocimiento propio de la dialéctica entre el amo y el esclavo, el cual es reconstruido de modo minucioso. En el marco de esta reconstrucción, el autor presenta una visión crítica de la interpretación tradicional en la medida en que la explica no como la confrontación de dos auto-conciencias, sino como dos perspectivas propias de la autoconciencia. Lo que el argumento logra, a fin de cuentas, es disolver el fondo problemático que da lugar a la antinomia. En cualquier caso, el texto tiene la virtud de colocar bajo una nueva luz, la del problema de la libertad cosmológica, el conocido capítulo de la Fenomenología del espíritu.

La edición concluye con "Does Spontaneity have to be Naturalized? Freedom as Spontaneity - Today and in Kant", escrito por Jakub KlocKonkolowicz- - Al hablar de espontaneidad naturalizada, el autor se refiere al significado antropológico o cotidiano que porta este concepto. La preponderancia de una visión antropológica es atribuida a autores contemporáneos como Arendt, Habermas y Nussbaum, pues interpretan la espontaneidad como un concepto fundamental en la filosofía moral, pero lo interpretan como una propiedad social que debe ser adquirida a través de un proceso de mediación colectivo. Pero por sobre la determinación social, que para el autor permanece siempre externa al actor, se encuentra la determinación individual. Y solo bajo esta determinación, la espontaneidad permanece como una posibilidad para todo sujeto, con independencia de su situación histórica concreta. Si la crítica de Kloc-Konkolowicz es correcta, es finalmente el concepto transcendental aquel que, en un sentido relevante, termina siendo el más inclusivo. De esta manera, el volumen consigue un cierre orgánico, pues además de haber reconstruido a lo largo de sus capítulos los distintos contextos históricos en los cuales el concepto de libertad cosmológica ha jugado un rol, nos permite evaluar incluso en qué sentidos puede aún participar en las discusiones más contemporáneas. 
Es posible que, al considerar el índice del volumen, el lector eche en falta un capítulo exclusivamente dedicado a la reconstrucción del tercer conflicto antinómico de la razón pura. Por el contrario, todos los capítulos, aún los de mayor pretensión sistemática, intentan situar al concepto de Kant en un diálogo con la tradición filosófica. Lo que esto significa, aunque sea implícitamente, es que nuestra apropiación del pensamiento kantiano ya no puede realizarse sin considerar la mediación del pensamiento post-kantiano. Podemos decir, como conclusión, que la armonía entre el desarrollo histórico y la sutileza de las reconstrucciones sistemáticas es lo que hace muy valioso a este volumen y que interesará por igual a quien indaga en aspectos técnicos del concepto moderno de libertad como a quien busca obtener una visión de conjunto del tema, tomando como punto focal, en este caso, la doctrina de Kant. 